\title{
HEISENBERG UNIQUENESS PAIRS IN THE PLANE. THREE PARALLEL LINES
}

\author{
DANIEL BLASI BABOT
}

(Communicated by Michael T. Lacey)

\begin{abstract}
A Heisenberg uniqueness pair is a pair $(\Gamma, \Lambda)$, where $\Gamma$ is a curve in the plane and $\Lambda$ is a set in the plane, with the following property: any bounded Borel measure $\mu$ in the plane supported on $\Gamma$, which is absolutely continuous with respect to arc length and whose Fourier transform $\widehat{\mu}$ vanishes on $\Lambda$, must automatically be the zero measure. We characterize the Heisenberg uniqueness pairs for $\Gamma$ as being three parallel lines $\Gamma=\mathbb{R} \times\{\alpha, \beta, \gamma\}$ with $\alpha<\beta<\gamma$, $(\gamma-\alpha) /(\beta-\alpha) \in \mathbb{N}$.
\end{abstract}

\section{INTRODUCTION}

The Heisenberg uncertainty principle states that both a function and its Fourier transform cannot be too localized at the same time (see [2] and [3]). M. Benedicks in [1] proved that given a nontrivial function $f \in L^{1}\left(\mathbb{R}^{n}\right)$, the Lebesgue measure of the set of points where $f \neq 0$ and the set of points where the Fourier transform $\widehat{f} \neq 0$ cannot be simultaneously finite. In this paper we consider a similar problem for measures supported on a subset of $\mathbb{R}^{2}$.

Let $\Gamma$ be a smooth curve in the plane $\mathbb{R}^{2}$ and $\Lambda$ a subset in $\mathbb{R}^{2}$. In [4, Hedenmalm and Montes-Rodríguez posed the problem of deciding when it is true that

$$
\widehat{\mu}_{\mid \Lambda}=0 \text { implies } \mu=0
$$

for any Borel measure $\mu$ supported on $\Gamma$ and absolutely continuous with respect to the arc length measure on $\Gamma$, where

$$
\widehat{\mu}(\xi, \eta)=\int_{\mathbb{R}^{2}} e^{\pi i\langle(x, y),(\xi, \eta)\rangle} d \mu(x, y) .
$$

If this is the case, then $(\Gamma, \Lambda)$ is called a Heisenberg Uniqueness Pair (HUP).

When $\Gamma$ is the circle, Lev [7] and Sjölin [8] independently characterized the HUP for some "small" sets $\Lambda$.

In [4] Hedenmalm and Montes-Rodríguez characterized the HUP in the cases:

- $\Gamma$ is the hyperbola $x y=1$ and $\Lambda=(\alpha \mathbb{Z} \times\{0\}) \cup(\{0\} \times \beta \mathbb{Z})$, for $\alpha, \beta>0$.

- $\Gamma$ two parallel lines in $\mathbb{R}^{2}$.

Received by the editors March 29, 2011 and, in revised form, October 29, 2011 and January 23, 2012 .

2010 Mathematics Subject Classification. Primary 42B10; Secondary 31B35, 81Q05.

Key words and phrases. Heisenberg uniqueness pairs, uncertainty principle.

The author was partially supported by the Göran Gustaffson Foundation, grant No. 2009SGR00420, and the DGICYT grant MTM2008-00145. 
In this note we present a result generalizing this last case. We characterize the HUP for $\Gamma$ as being three parallel lines:

$$
\Gamma=\mathbb{R} \times\{\alpha, \beta, \gamma\} \text { with } \alpha<\beta<\gamma,(\gamma-\alpha) /(\beta-\alpha) \in \mathbb{N} .
$$

\section{Three PARALLEL LINES}

Given a set $E \subset \mathbb{R}$ and a point $\xi \in E$, let us define:

- $\mathcal{A}_{\text {loc }}^{E, \xi}=\left\{\right.$ functions $\psi$ defined on $E$ such that there exist a small interval $I_{\xi}$ around $\xi$ and a function $\varphi \in L^{1}(\mathbb{R})$ such that $\psi(\zeta)=\widehat{\varphi}(\zeta)$, for $\left.\zeta \in I_{\xi} \cap E\right\}$.

- $P^{1, p}\left[\mathcal{A}_{\text {loc }}^{E, \xi}\right]=\left\{\right.$ functions $\psi$ defined on $E$ such that there exist an interval $I_{\xi}$ around $\xi$ and functions $\varphi_{0}, \varphi_{1} \in L^{1}(\mathbb{R})$ with $\psi^{p}(\zeta)+\widehat{\varphi}_{1}(\zeta) \psi(\zeta)+\widehat{\varphi}_{0}(\zeta)=0$, for $\left.\zeta \in I_{\xi} \cap E\right\}$.

Wiener's lemma [5. p. 57] states that if $\psi \in \mathcal{A}_{l o c}^{E, \xi}$ and $\psi(\xi) \neq 0$, then $1 / \psi \in$ $\mathcal{A}_{l o c}^{E, \xi}$. Observe also that if $\psi \in \mathcal{A}_{l o c}^{E, \xi}$, then $\psi \in P^{1, p}\left[\mathcal{A}_{l o c}^{E, \xi}\right]$. This is easy to see only if $p$ is natural.

Due to invariance under translation and rescaling (see [4]) it will be sufficient to study the case when $\Gamma=\mathbb{R} \times\{0,1, p\}$ for $p \in \mathbb{N}, p>1$.

Given a set $\Lambda \subset \mathbb{R}^{2}$, we say that $\mu$ is an admissible measure if $\mu$ is a Borel measure in the plane absolutely continuous with respect to arc length with supp $\mu \subset \Gamma$ and $\widehat{\mu}_{\mid \Lambda}=0$.

If $\mu$ is a measure absolutely continuous with respect to arc length on $\Gamma$, then there exist functions $f, g, h \in \mathrm{E}^{1}(\mathbb{R})$ such that

$$
\widehat{\mu}(\xi, \eta)=\widehat{f}(\xi)+e^{\pi i \eta} \widehat{g}(\xi)+e^{p \pi i \eta} \widehat{h}(\xi), \text { for any }(\xi, \eta) \in \mathbb{R}^{2} .
$$

In particular an admissible measure can be written in this form. Observe also that $\widehat{\mu}$ is 2 -periodic with respect to the second variable. So, for any set $\Lambda \subset \mathbb{R}^{2}$, we may consider the periodized set

$$
\mathcal{P}(\Lambda)=\{(\xi, \eta) \text { such that }(\xi, \eta+2 k) \in \Lambda \text { for some } k \in \mathbb{Z}\},
$$

and it follows that $(\Gamma, \Lambda)$ is a HUP if and only if $(\Gamma, \overline{\mathcal{P}(\Lambda)})$ is a HUP, where $\overline{\mathcal{P}(\Lambda)}$ stands for the closure of $\mathcal{P}(\Lambda)$ in $\mathbb{R}^{2}$.

We may think without loss of generality that $\Lambda$ is a closed set in $\mathbb{R}^{2}, 2$-periodic with respect to the second coordinate.

We then have the following result.

Theorem 1. Let $\Gamma=\mathbb{R} \times\{0,1, p\}$, for some $p \in \mathbb{N}, p>1$ and $\Lambda \subset \mathbb{R}^{2}$, closed and 2-periodic with respect to the second variable. Then $(\Gamma, \Lambda)$ is a Heisenberg uniqueness pair if and only if

$$
\mathfrak{F}:=\Pi^{3}(\Lambda) \cup\left(\Pi^{2}(\Lambda) \backslash \Pi^{2^{*}}(\Lambda)\right) \cup\left(\Pi^{1}(\Lambda) \backslash \Pi^{1^{*}}(\Lambda)\right)
$$

is dense in $\mathbb{R}$.

$\Pi(\Lambda)$ means the projection of $\Lambda$ on the axis $\mathbb{R} \times\{0\}$ and given a point $\xi \in \Pi(\Lambda)$, and $\operatorname{Img}(\xi)$ corresponds to the set of points $\eta \in[0,2)$ with $(\xi, \eta) \in \Lambda$. The sets in $\mathfrak{F}$ are defined as follows:

- $\Pi^{1}(\Lambda)=\left\{\xi \in \Pi(\Lambda)\right.$ such that there is a unique $\left.\eta_{0} \in \operatorname{Img}(\xi)\right\}$.

- $\Pi^{2}(\Lambda)=\left\{\xi \in \Pi(\Lambda)\right.$ such that there are two different points $\eta_{0}, \eta_{1} \in$ $\operatorname{Img}(\xi)$, and if there is another point $\eta_{2} \in \operatorname{Im} g(\xi)$, then $\frac{e^{p \pi i \eta_{1}}-e^{p \pi i \eta_{0}}}{e^{\pi i \eta_{1}}-e^{\pi i \eta_{0}}}=$ $\left.\frac{e^{p \pi i \eta_{2}}-e^{p \pi i \eta_{0}}}{e^{\pi i \eta_{2}}-e^{\pi i \eta_{0}}}\right\}$ 
- $\Pi^{3}(\Lambda)=\{\xi \in \Pi(\Lambda)$ such that there are at least three different points $\eta_{0}, \eta_{1}, \eta_{2} \in \operatorname{Img}(\xi)$ with $\left.\frac{e^{p \pi i \eta_{1}}-e^{p \pi i \eta_{0}}}{e^{\pi i \eta_{1}}-e^{\pi i \eta_{0}}} \neq \frac{e^{p \pi i \eta_{2}}-e^{p \pi i \eta_{0}}}{e^{\pi i \eta_{2}}-e^{\pi i \eta_{0}}}\right\}$.

The following technical lemma is easy to prove and shows that the functions $\tau$ and $\Phi$ are well defined for $\xi \in \Pi^{2}(\Lambda)$.

Lemma 2. Let $x, y, z \in \mathbb{C}$ be different with

$$
\tau=\frac{y^{p}-x^{p}}{y-x}=\frac{z^{p}-x^{p}}{z-x}
$$

then

$$
\frac{z^{p}-y^{p}}{z-y}=\tau \quad \text { and } \quad \Phi=x \tau-x^{p}=y \tau-y^{p}=z \tau-z^{p} .
$$

Let $\chi$ be a function defined on $\Pi^{1}(\Lambda)$ as $\chi(\zeta)=e^{\pi i \eta}$, where $\eta \in \operatorname{Img}(\zeta)$. We define the set $\Pi^{1^{*}}(\Lambda)$ as

- $\Pi^{1^{*}}(\Lambda)=\left\{\xi \in \Pi^{1}(\Lambda)\right.$ such that $\left.\chi \in P^{1, p}\left[\mathcal{A}_{l o c}^{\Pi^{1}(\Lambda), \xi}\right]\right\}$.

Let $\tau, \Phi$ be functions defined on $\Pi^{2}(\Lambda)$ as

$$
\tau(\xi)=\frac{e^{p \pi i \eta_{1}}-e^{p \pi i \eta_{0}}}{e^{\pi i \eta_{1}}-e^{\pi i \eta_{0}}} \quad \text { and } \quad \Phi(\xi)=e^{\pi i \eta_{0}} \frac{e^{p \pi i \eta_{1}}-e^{p \pi i \eta_{0}}}{e^{\pi i \eta_{1}}-e^{\pi i \eta_{0}}}-e^{p \pi i \eta_{0}},
$$

where $\eta_{0}, \eta_{1} \in \operatorname{Img}(\xi)$. We define the set $\Pi^{2^{*}}(\Lambda)$ as

- $\Pi^{2^{*}}(\Lambda)=\left\{\xi \in \Pi^{2}(\Lambda)\right.$ such that $\left.\tau, \Phi \in \mathcal{A}_{\text {loc }}^{\Pi^{2}(\Lambda), \xi}\right\}$.

The next lemma will be needed for the proof of the necessity of condition (2.1) in Theorem 1 .

Lemma 3. Let $I$ be an interval in $\mathbb{R}$ with $\Pi^{2^{*}}(\Lambda)$ dense in $I$. Then there exists a subinterval $I^{\prime} \subset I$ with $I^{\prime} \subset \Pi^{2^{*}}(\Lambda) \cup \Pi^{3}(\Lambda)$.

Proof. Pick an arbitrary point $\widetilde{\xi} \in I \cap \Pi^{2^{*}}(\Lambda)$. Since $\tau, \Phi \in \mathcal{A}_{l o c}^{\Pi^{2}(\Lambda), \widetilde{\xi}}$ and $\Pi^{2^{*}}(\Lambda)$ is dense in $I$, we can extend the functions $\tau, \Phi$ continuously on a neighborhood of $\widetilde{\xi}$. Let $\widetilde{\eta} \neq \widetilde{\varrho} \in \operatorname{Img}(\widetilde{\xi})$. Then

$$
|\tau(\widetilde{\xi})|=\left|\frac{e^{p \pi i \widetilde{\eta}}-e^{p \pi i \widetilde{\varrho}}}{e^{\pi i \widetilde{\eta}}-e^{\pi i \widetilde{\varrho}}}\right|<p,
$$

and since $\tau$ is continuous around $\widetilde{\xi}$, there exists a small interval $I^{\prime}$ around $\widetilde{\xi}$ with $|\tau(\xi)|<p$ for $\xi \in I^{\prime}$. We will see that $I^{\prime} \subset \Pi^{2^{*}}(\Lambda) \cup \Pi^{3}(\Lambda)$.

Given $\xi \in I^{\prime}$, consider a sequence $\left\{\xi_{k}\right\} \subset \Pi^{2^{*}}(\Lambda) \cap I^{\prime}$ with $\xi_{k} \rightarrow \xi$, and for each $\xi_{k}$ let $\eta_{k} \neq \varrho_{k} \in \operatorname{Im} g\left(\xi_{k}\right)$. There exist subsequences $\left\{\eta_{k}^{*}\right\}$ and $\left\{\varrho_{k}^{*}\right\}$ such that $\eta_{k}^{*} \rightarrow \eta^{*}$ and $\varrho_{k}^{*} \rightarrow \varrho^{*}$ for some $\eta^{*}, \varrho^{*} \in[0,2]$. Since the set $\Lambda$ is closed and 2-periodic with respect to the second coordinate, we may assume WLOG that $\xi \in \Pi(\Lambda)$ with $\eta^{*} \neq \varrho^{*} \in \operatorname{Img}(\xi)$. Otherwise,

$$
\begin{aligned}
|\tau(\xi)| & \longleftarrow\left|\tau\left(\xi_{k}^{*}\right)\right|=\left|e^{(p-1) \pi i \eta_{k}^{*}}+e^{(p-2) \pi i \eta_{k}^{*}} e^{\pi i \varrho_{k}^{*}}+\cdots+e^{(p-1) \pi i \varrho_{k}^{*}}\right| \\
& \longrightarrow\left|e^{(p-1) \pi i \eta^{*}}+e^{(p-1) \pi i \eta^{*}}+\cdots+e^{(p-1) \pi i \eta^{*}}\right|=p
\end{aligned}
$$

which is a contradiction with the fact that $\xi \in I^{\prime}$.

So $I^{\prime} \subset \Pi^{2}(\Lambda) \cup \Pi^{3}(\Lambda)$, and since the extended functions $\tau, \Phi$ are continuous on $I^{\prime}$, we also have that $\xi \in \Pi^{2^{*}}(\Lambda)$ for any $\xi \in \Pi^{2}(\Lambda) \cap I^{\prime}$. Also, we can conclude that $I^{\prime} \subset \Pi^{2^{*}}(\Lambda) \cup \Pi^{3}(\Lambda)$. 


\section{Proof of the MAIN RESUlt}

This section is devoted to the proof of Theorem 1. The proof of the sufficiency of condition (2.1) is rather easy. Let $\mu$ be an admissible measure. Then there exist functions $f, g, h \in \mathrm{E}^{1}(\mathbb{R})$ such that

$$
\widehat{\mu}(\xi, \eta)=\widehat{f}(\xi)+e^{\pi i \eta} \widehat{g}(\xi)+e^{p \pi i \eta} \widehat{h}(\xi), \text { for any }(\xi, \eta) \in \mathbb{R}^{2} .
$$

Since $\mathfrak{F}$ is dense in $\mathbb{R}$ we will be done if we show that $\widehat{f}(\xi)=\widehat{g}(\xi)=\widehat{h}(\xi)=0$ for any $\xi \in \mathfrak{F}=\Pi^{3}(\Lambda) \cup\left(\Pi^{2}(\Lambda) \backslash \Pi^{2^{*}}(\Lambda)\right) \cup\left(\Pi^{1}(\Lambda) \backslash \Pi^{1^{*}}(\Lambda)\right)$.

If $\xi \in \Pi^{3}(\Lambda)$, let $\eta_{0}, \eta_{1}, \eta_{2} \in \operatorname{Img}(\xi)$ be different. Since $\widehat{\mu}_{\mid \Lambda}=0$ and $\frac{e^{p \pi i \eta_{1}}-e^{p \pi i \eta_{0}}}{e^{\pi i \eta_{1}}-e^{\pi i \eta_{0}}}$ $\neq \frac{e^{p \pi i \eta_{2}}-e^{p \pi i \eta_{0}}}{e^{\pi i \eta_{2}}-e^{\pi i \eta_{0}}}$, it follows that $\widehat{f}(\xi)=\widehat{g}(\xi)=\widehat{h}(\xi)=0$.

If $\xi \in \Pi^{2}(\Lambda)$, let $\eta_{0} \neq \eta_{1} \in \operatorname{Img}(\xi)$. Since $\widehat{\mu}_{\mid \Lambda}=0$, then $\widehat{g}(\xi)=-\tau(\xi) \widehat{h}(\xi)$ and $\widehat{f}(\xi)=\Phi(\xi) \widehat{h}(\xi)$. Suppose $\widehat{h}(\xi) \neq 0$. Then by Wiener's lemma and Fubini's theorem, $\tau, \Phi \in \mathcal{A}_{l o c}^{\Pi^{2}(\Lambda), \xi}$, which implies that $\xi \in \Pi^{2^{*}}(\Lambda)$. So if $\xi \in \Pi^{2}(\Lambda) \backslash \Pi^{2^{*}}(\Lambda)$, then $\widehat{f}(\xi)=\widehat{g}(\xi)=\widehat{h}(\xi)=0$.

Finally, if $\xi \in \Pi^{1}(\Lambda)$ and $\eta_{0} \in \operatorname{Img}(\xi)$, since $\widehat{\mu}_{\mid \Lambda}=0$, then $\widehat{f}(\xi)+\chi(\xi) \widehat{g}(\xi)+$ $\chi^{p}(\xi) \widehat{h}(\xi)=0$, where $\chi(\xi)=e^{\pi i \eta_{0}}$. Suppose $\widehat{h}(\xi) \neq 0$; then $\chi \in P^{1, p}\left[\mathcal{A}_{l o c}^{\Pi^{1}(\Lambda), \xi}\right]$ and $\xi \in \Pi^{1^{*}}(\Lambda)$. Otherwise, if $\widehat{h}(\xi)=0$ and $\widehat{g}(\xi) \neq 0$, then by Wiener's lemma and Fubini's theorem, $\chi \in \mathcal{A}_{l o c}^{\Pi^{1}(\Lambda), \xi}$ and also $\chi^{p} \in \mathcal{A}_{l o c}^{\Pi^{1}(\Lambda), \xi}$, so $\chi \in P^{1, p}\left[\mathcal{A}_{l o c}^{\Pi^{1}(\Lambda), \xi}\right]$ and $\xi \in \Pi^{1^{*}}(\Lambda)$. This means that if $\xi \in \Pi^{1}(\Lambda) \backslash \Pi^{1^{*}}(\Lambda)$, then $\widehat{f}(\xi)=\widehat{g}(\xi)=\widehat{h}(\xi)=0$.

For the proof of the necessity of condition (2.1), suppose that the set $\mathfrak{F}$ is not dense in $\mathbb{R}$ and let us pick an open interval $I$ that has empty intersection with $\mathfrak{F}$, i.e.,

$$
\Pi(\Lambda) \cap I=\left(\Pi^{1^{*}}(\Lambda) \cup \Pi^{2^{*}}(\Lambda)\right) \cap I .
$$

We consider three cases:

- There exists a small interval $I_{\xi} \subset I$ around $\xi \in \Pi^{1^{*}}(\Lambda)$ such that all the points in $I_{\xi} \cap \Pi(\Lambda)$ belong to $\Pi^{1^{*}}(\Lambda)$. Since $\chi \in P^{1, p}\left[\mathcal{A}_{l o c}^{\Pi^{1}(\Lambda), \xi}\right]$, there exist an interval $I^{\prime} \subset I_{\xi}$ around $\xi$ and functions $\varphi_{0}, \varphi_{1} \in L^{1}(\mathbb{R})$ such that

$$
\chi^{p}\left(\xi^{*}\right)+\widehat{\varphi}_{1}\left(\xi^{*}\right) \chi\left(\xi^{*}\right)+\widehat{\varphi}_{0}\left(\xi^{*}\right)=0
$$

for any $\xi^{*} \in I^{\prime} \cap \Pi(\Lambda)$. Let $h \in L^{1}(\mathbb{R})$ with $\widehat{h}(\xi) \neq 0$ and supp $\widehat{h} \Subset I^{\prime}$, and define $f, g \in L^{1}(\mathbb{R})$ via $\widehat{f}=\widehat{h} \widehat{\varphi_{0}}$, and $\widehat{g}=\widehat{h} \widehat{\varphi_{1}}$. Now,

$$
\widehat{\mu}\left(\xi^{*}, \eta^{*}\right)=\widehat{f}\left(\xi^{*}\right)+\widehat{g}\left(\xi^{*}\right) \chi\left(\xi^{*}\right)+\widehat{h}\left(\xi^{*}\right) \chi^{p}\left(\xi^{*}\right)=0
$$

for $\xi^{*} \in I^{\prime} \cap \Pi^{1^{*}}(\Lambda), \eta^{*} \in \operatorname{Img}\left(\xi^{*}\right)$. Finally, since supp $\widehat{h} \Subset I^{\prime}$ and $I^{\prime} \cap$ $\Pi(\Lambda)=I^{\prime} \cap \Pi^{1^{*}}(\Lambda)$, we can conclude that $\widehat{\mu}_{\left.\right|_{\Lambda}} \equiv 0$, and we have that $\mu$ is a nontrivial admissible measure. So $(\Gamma, \Lambda)$ is not a Heisenberg uniqueness pair.

- There exists a small interval $I_{\xi} \subset I$ around $\xi \in \Pi^{2^{*}}(\Lambda)$ such that all the points in $I_{\xi} \cap \Pi(\Lambda)$ belong to $\Pi^{2^{*}}(\Lambda)$. Now there exists a small interval $I^{\prime} \subset I_{\xi}$ around $\xi$ and functions $\Phi_{1}, \tau_{1} \in L^{1}(\mathbb{R})$ such that $\widehat{\tau_{1}}=\tau$ and $\widehat{\Phi_{1}}=\Phi$ on $I^{\prime} \cap \Pi(\Lambda)$. Consider a function $h \in L^{1}(\mathbb{R})$ with supp $\widehat{h} \subset I^{\prime}$ and $\widehat{h}(\xi) \neq 0$, and define $f, g \in L^{1}(\mathbb{R})$ as

$$
g=-h * \tau_{1} \quad \text { and } \quad f=h * \Phi_{1} .
$$


Now, given a point $\xi^{*} \in I^{\prime} \cap \Pi^{2^{*}}(\Lambda)$, let $\eta^{*} \neq \varrho^{*} \in \operatorname{Img}\left(\xi^{*}\right)$. Since $\tau\left(\xi^{*}\right)=$ $\frac{e^{p \pi i \eta^{*}}-e^{p \pi i \varrho^{*}}}{e^{\pi i \eta^{*}}-e^{\pi i \varrho^{*}}}$ and $\Phi(\xi)=e^{\pi i \eta^{*}} \frac{e^{p \pi i \eta^{*}}-e^{p \pi i \varrho^{*}}}{e^{\pi i \eta^{*}}-e^{\pi i \varrho^{*}}}-e^{p \pi i \varrho^{*}}$, we have that

$$
\widehat{\mu}\left(\xi^{*}, \eta^{*}\right)=\widehat{f}\left(\xi^{*}\right)+\widehat{g}\left(\xi^{*}\right) e^{\pi i \eta^{*}}+\widehat{h}\left(\xi^{*}\right) e^{p \pi i \eta^{*}}=0
$$

and also that $\widehat{\mu}\left(\xi^{*}, \varrho^{*}\right)=0$. So, the corresponding measure $\mu$ is a nontrivial admissible measure and $(\Gamma, \Lambda)$ is not a Heisenberg uniqueness pair.

- All the intervals $I_{\xi} \subset I$ contain points in $\Pi^{1^{*}}(\Lambda)$ and points in $\Pi^{2^{*}}(\Lambda)$. That is, the sets $\Pi^{1^{*}}(\Lambda)$ and $\Pi^{2^{*}}(\Lambda)$ are dense in $I \cap\left(\Pi^{1^{*}}(\Lambda) \cup \Pi^{2^{*}}(\Lambda)\right)=I \cap \Pi(\Lambda)$. But this is not possible. In fact, if $\Pi^{2^{*}}(\Lambda)$ is dense in $I$, by Lemma 3 , there exists a subinterval $I^{\prime} \subset I$ such that $I^{\prime} \subset \Pi^{2^{*}}(\Lambda) \cup \Pi^{3}(\Lambda)$.

This finishes the proof of the theorem.

\section{EXAmples AND FURTHER RESUlts}

Given a point $\xi \in \Pi(\Lambda)$ such that $\sharp\{\eta \in \operatorname{Img}(\xi)\} \geq 3$, we will state a criteria to decide whether the point $\xi$ belongs to $\Pi^{3}(\Lambda)$ or to $\Pi^{2}(\Lambda)$. But before this we prove the following lemma.

Lemma 4. Given $C \in \mathbb{C}$, there exist at most $p$ different points $\rho^{(k)} \in[0,2)$ such that for any $j \neq k$,

$$
\frac{x^{p}-y^{p}}{x-y}=C, \text { where } x=e^{\pi i \rho^{(k)}}, y=e^{\pi i \rho^{(j)}} .
$$

Proof. Observe that for fixed $C$, there exists a constant $C^{*} \in \mathbb{C}$ such that

$$
x C-x^{p}=C^{*}
$$

for any $x=e^{\pi i \rho^{(k)}}$ solution of (4.1). Now it is obvious that there are at most $p$ different solutions $\rho^{(k)} \in[0,2)$ of the equation (4.2).

Corollary 5. Given a point $\xi \in \Pi(\Lambda)$, if $\sharp\{\eta \in \operatorname{Img}(\xi)\}>p$, then $\xi \in \Pi^{3}(\Lambda)$.

In particular, if $\Gamma$ consists of three parallel equidistant lines in the plane $(p=2)$, we have

$$
\begin{aligned}
& \Pi^{3}(\Lambda)=\{\xi \in \Lambda \text { such that } \sharp\{\eta \in \operatorname{Img}(\xi)\} \geq 3\}, \\
& \Pi^{2}(\Lambda)=\{\xi \in \Lambda \text { such that } \sharp\{\eta \in \operatorname{Img}(\xi)\}=2\} .
\end{aligned}
$$

Example 6. The following example shows that Corollary 5 is sharp:

- Let $\Lambda=\mathbb{R} \times\{2 k / p\}_{k=0, \cdots, p-1}$. Then for any $\xi \in \mathbb{R}$,

$$
\sharp\{\eta \in \operatorname{Img}(\xi)\}=p
$$

and $\xi \in \Pi^{2^{*}}(\Lambda)$. Observe that in this case, $(\Gamma, \Lambda)$ is not an HUP.

This lemma will be useful for another example.

Lemma 7. For any $z \in \mathbb{C}$ with $|z|<1$, there exist $w_{1}, w_{2} \in \mathbb{C}$ unimodular with $z=w_{1}+w_{2}$.

Proof. Let $z=r e^{i \sigma}$ and let $v \in[0, \pi / 2]$ with $\cos v=r / 2$. Let's take

$$
w_{1}=e^{i(v+\sigma)}, \quad w_{2}=e^{i(-v+\sigma)} .
$$

Then,

$$
w_{1}+w_{2}=e^{i(v+\sigma)}+e^{i(-v+\sigma)}=e^{i \sigma} 2 \cos (v)=r e^{i \sigma}=z,
$$

and this finishes the proof. 
Example 8. Let $p=2$. Let $g$ be a bounded, continuous function with $|g|<1$ that is nowhere locally the Fourier transform of an $L^{1}$ function. There exists a set $\Lambda \subset \mathbb{R} \times[0,2)$ such that $\Pi(\Lambda)=\Pi^{2^{*}}(\Lambda)$ is dense in $\mathbb{R}$ and the function $\Phi \equiv g$ on $\Pi^{2^{*}}(\Lambda)$. So, $(\Gamma, \Lambda)$ is not an HUP.

Let's first prove the existence of the function $g$. Let $E$ be a dense set of measure zero on the circle $\mathbb{T}$. By [6] there exists a continuous function $f$ such that the Fourier series of $f$ fails to converge on any point of $E$. Now let $g: \mathbb{R} \rightarrow \mathbb{C}$ be the 2-periodic function defined as $g(t)=f\left(e^{\pi i t}\right)$. It is easy to see that this function $g$ is continuous but it is not a Fourier transform of an $L^{1}$ function locally at any point. By a standard argument we can think that $g$ is bounded with $|g|<1$.

Now we will define the set $\Lambda$. By Lemma 7 , for any $\xi \in \mathbb{R}$ there exist $w_{1}(\xi)=$ $e^{\pi i \eta_{0}}, w_{2}(\xi)=e^{\pi i \eta_{1}}$ with $w_{1}(\xi)+w_{2}(\xi)=g(\xi)$. Observe also that there is a dense set $\Psi$ of $\mathbb{R}$ such that $\eta_{0} \neq \eta_{1}$ for any $\xi \in \Psi$. Otherwise the function $g$ is constant on an interval, and we get a contradiction with the fact that $g$ is not locally the Fourier transform of an $L^{1}$ function.

We define $\Lambda=\left\{\left(\xi, \eta_{0}\right) \cup\left(\xi, \eta_{1}\right)\right\}_{\xi \in \Psi}$. Now $\Pi(\Lambda)=\Pi^{2}(\Lambda)$ and

$$
\Phi(\xi)=e^{\pi i \eta_{0}}+e^{\pi i \eta_{1}}=g(\xi) \text {, for any } \xi \in \Psi .
$$

Since $\Phi \notin \mathcal{A}_{l o c}^{\Pi^{2}(\Lambda), \xi}$ for any $\xi \in \Pi^{2}(\Lambda)$, we have that $\Pi(\Lambda)=\Pi^{2^{*}}(\Lambda)$, and so $(\Gamma, \Lambda)$ is not an HUP.

\section{ACKNOWLEDGEMENTS}

The author thanks Håkan Hedenmalm for proposing the problem and Ioannis Parissis and Joaquim Ortega-Cerdà for helpful comments.

\section{REFERENCES}

[1] M. Benedicks, On Fourier transforms of functions supported on sets of finite Lebesgue measure, J. Math. Anal. Appl. 106 (1985), no. 1, 180-183. MR780328 (86f:43006)

[2] W. Heisenberg, Uber den anschaulichen Inhalt der quantentheoretischen Kinematik und Mechanik, Physik 43 (1927), 172-198.

[3] V. Havin; B. Jöricke, The uncertainty principle in harmonic analysis, Ergebnisse der Mathematik und ihrer Grenzgebiete (3) [Results in Mathematics and Related Areas (3)], 28. Springer-Verlag, Berlin, 1994. MR,1303780 (96c:42001)

[4] H. Hedenmalm; A. Montes-Rodríguez, Heisenberg uniqueness pairs and the Klein-Gordon equation, Ann. of Math. (2) 173 (2011), no. 3, 1507-1527. MR2800719

[5] J.P. Kahane, Séries de Fourier absolument convergentes, Ergebnisse der Mathematik und ihrer Grenzgebiete, Band 50, Springer-Verlag, Berlin-New York (1970). MR0275043 (43:801)

[6] J.P. Kahane; Y. Katznelson, Sur les ensembles de divergence des séries trigonométriques, Studia Math. 26 (1966), 305-306. MR0199633 (33:7776)

[7] N. Lev, Uniqueness theorems for Fourier transforms, Bull. Sci. Math. 135 (2011), no. 2, 134-140. MR2773393

[8] P. Sjölin, Heisenberg uniqueness pairs and a theorem of Beurling and Malliavin, Bull. Sci. Math. 135 (2011), no. 2, 125-133. MR2773392(2012c:42026)

Departament de Matemàtiques, Universitat Autònoma de Barcelona, 08193 Cerdanyola del Vallès, Catalunya, Spain

E-mail address: dblasi@gmail.com 